\title{
Unveiled optical properties of tetrapyrollic pigments in cryogenic environments
}

\author{
C. Crépin, N. Shafizadeh, and W. Chin
}

Institut des Sciences Moléculaires d'Orsay, Bât. 210

J.-P. Galaup ${ }^{\text {a) }}$

Laboratoire Aimé Cotton, Bât. 505 CNRS \& Université Paris-Sud 11, 91405 Orsay cedex, France

\section{J. G. McCaffrey}

Department of Chemistry, National University of Ireland, Maynooth, Ireland

\section{S. M. Arabei}

Belarusian State Agrarian and Technical University, Minsk, Belarus

(Submitted February 15, 2010)

Fiz. Nizk. Temp. 36, 563-570 (May 2010)

\begin{abstract}
An unexpected phenomenon was revealed in the laser induced fluorescence spectra of free-base $\left(\mathrm{H}_{2} \mathrm{Pc}\right)$ and zinc $(\mathrm{ZnPc})$ phthalocyanines trapped in rare gas and nitrogen matrices under a moderate increase in the laser intensity. In all matrices the intensity of an emission band near $755 \mathrm{~nm}$ increased drastically when pumping the $S_{1} \leftarrow S_{0}$ transition. This observation was assigned to stimulated emission in a four-level scheme involving a vibronic transition from the lowest electronic state to a vibrational level of the ground state. In the present work, we expose new similar results obtained with porphyrin molecules, i.e. tetra-benzoporphin (TBP). With free-base $\mathrm{H}_{2} \mathrm{TBP}$, stimulated emission was observed in Ar or $\mathrm{N}_{2}$ matrices, but not in Xe matrices. A possible reason could be a fast inter-system crossing rate due to the heavy atom effect induced by Xe. We also report the observation of persistent burnt spectral holes, although the low efficiency of this process is not competitive with stimulated emission and no decrease of the stimulated emission with time was observed. With ZnTBP no stimulated emission could be recorded but the appearance of rather strong phosphorescence was noticed. Similar studies focusing on the stimulated emission phenomenon is that they should allow new insights into site effects and site selectivity. (C) 2010 American Institute of Physics. [doi:10.1063/1.3432263]
\end{abstract}

\section{INTRODUCTION}

Recent luminescence work $^{1}$ on phthalocyanine-doped cryogenic matrices revealed the occurrence of stimulated emission for specific vibronic transitions. This phenomenon was characterized by a drastic intensity increase of a specific vibronic fluorescence line with only a moderate increase of the laser pump intensity. The most concerned transition was assigned as the one taking place between the first excited electronic state and a vibrational level of the ground state located at approximately 1550 and $1525 \mathrm{~cm}^{-1}$ in $\mathrm{H}_{2} \mathrm{Pc}$ and ZnPc, respectively. ${ }^{1}$

One important question to address in the development of this work is whether the stimulated emission observed for the phthalocyanines in cryogenic matrices is a more general behavior occurring for other molecules in other environments. With this question in mind we are therefore in search for other systems, molecules and/or matrices, in which similar behavior might be observed. In present paper, we are looking for similar processes in the case of porphyrin molecules belonging to the tetrabenzoporphyrin family. Indeed, tetrabenzoporphin (TBP) molecules have the closest structure to the phthalocyanines $(\mathrm{Pc})$, the most noticeable change being the replacement of the four bridging nitrogen atoms belonging to the internal ring by carbon atoms (Fig. 1). Therefore similar vibronic structures are expected between both species.
The first absorption spectra of matrix-isolated porphyrins and phthalocyanines were reported early by Bajema et al. ${ }^{2,3}$ More recently Waluk and co-workers reported on the spectroscopy ${ }^{4}$ and isolation ${ }^{5}$ of porphyrins and related molecules in rare gas matrices. Other groups ${ }^{6-8}$ analyzed the vibronic structure on the $S_{1}$ state of several porphyrins and phthalocyanines in Shpol'skii matrices. A complete spectroscopic study and vibrational analysis of $\mathrm{H}_{2} \mathrm{TBP}$ in a Shpol'skii matrix of $n$-octane $\left(n-\mathrm{C}_{8}\right)$ was early reported by Arabei. ${ }^{9}$ The same author also studied the influence of temperature on the electronic spectra of $\mathrm{H}_{2} \mathrm{TBP}$ in $n$-octane. ${ }^{10}$ However, the behavior of this important class of molecules isolated in the solid rare gases needs still to be more thoroughly analyzed and characterized.

Laser-induced fluorescence spectroscopy is very well adapted for studying these molecules because most of their absorption bands in the visible region closely match the working range of tunable dye lasers. While undertaking such a spectroscopic study of matrix-isolated free-base phthalocyanine $\left(\mathrm{H}_{2} \mathrm{Pc}\right)$ and zinc phthalocyanine $(\mathrm{ZnPc})$, by means of emission-excitation spectra using pulsed dye lasers for excitation, an unusually intense vibronic band was observed with slightly increased laser power. This novel solid state effect, which had never been reported before, was the subject of our paper. ${ }^{1}$ The band involved corresponded to the transition from the first excited electronic state to a vibrational level of 


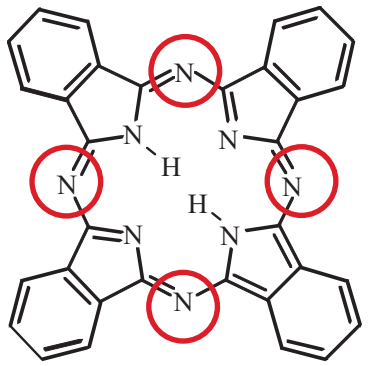

Free-base phthalocyanine $\left(\mathrm{H}_{2} \mathrm{Pc}\right)$

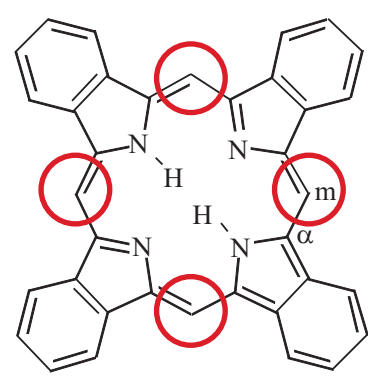

Tetrabenzoporphin $\left(\mathrm{H}_{2} \mathrm{TBP}\right)$
FIG. 1. Molecular structure of free-base phthalocyanine, as compared to that of free-base tetrabenzoporphin. The most noticeable difference between these two molecules involves replacement of the four bridging carbon atoms by nitrogen ones, as highlighted by the circles.

the ground state at approximately $1550 \mathrm{~cm}^{-1}$. The drastic intensity enhancement was attributed to the occurrence of stimulated emission (SE) on the basis of the following observations: 1) the laser power dependence of the phenomenon exhibits a well-defined threshold, 2) there is also a threshold as a function of the concentration of dopants, 3) the emission band is narrow compared to the spectral width of the standard fluorescence, even in the case of broad bands in the absorption spectrum, and 4) its lifetime is strongly shortened in comparison with other vibronic transitions of the emission spectrum and exhibits a perfect synchronism with the exciting laser pulses. ${ }^{1}$

A complete analysis is complicated by the different possible sites occupied by the guest molecules in the crystalline host; however, one important point noticed was that the SE line appeared as a narrow line from the most populated family of sites. In the case of $\mathrm{H}_{2} \mathrm{Pc} / \mathrm{N}_{2}$, where different sites were clearly observed in the absorption band, SE exhibited several lines, correlated with the main sites. Excitation spectra highlighted energy transfers between the families of sites. In the case of $\mathrm{H}_{2} \mathrm{Pc} / \mathrm{Ne}$, a spectral hole burning ( $\mathrm{SHB}$ ) phenomenon was also present and competed with SE, resulting in a decrease of the SE intensity with the total irradiation time. ${ }^{1}$ These studies of the SE phenomenon should allow new insights into site effects and site selectivity. Possible reasons for the different observations made depending on the different hosts studied have still to be discussed. To extend the range of guest molecules beyond those belonging to the phthalocyanine family, it is easy to be convinced of the strong similarity, both in terms of the molecular structure and the spectroscopy, between phthalocyanines and tetrabenzoporphyrins (Fig. 1). The aim of the present paper is to present new and preliminary results obtained with molecules belonging to benzoporphyrins in different cryogenic environments.

\section{EXPERIMENTAL DETAILS}

The tetrabenzoporphyrins, free-base $\mathrm{H}_{2} \mathrm{TBP}$ as well as ZnTBP, studied in this work were not commercial products. However, we can mention that Frontier Scientific Inc. can also make these molecules available. The molecules we used were synthesized according to the procedure described in

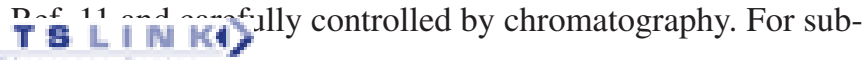

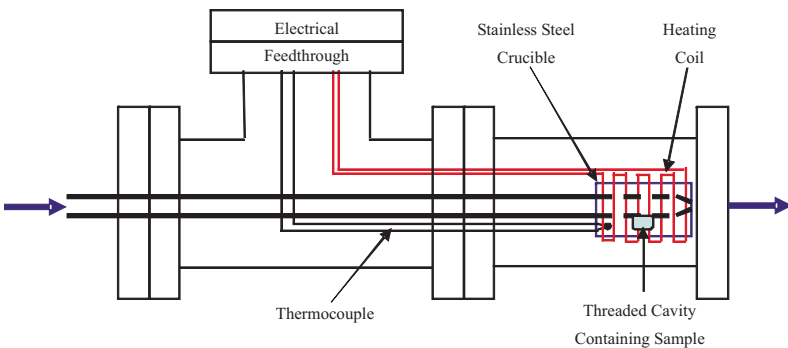

FIG. 2. Simplified scheme of the oven, especially designed by Dr. Michel Broquier and Dr. Pierre Çarçabal, for introducing dye molecules into the gas flow for deposition on a cooled glass substrate.

limation of the dye powder a specially designed oven, whose schematic is shown in Fig. 2, was used in the experiments. It consisted of a solid, stainless steel cylinder into which a hollow screw containing the dye powder was fitted. The top of this screw was positioned to emerge at right angles to a $2 \mathrm{~mm}$ opening passing through the length of the cylinder. This opening was connected to the gas inlet line. Resistive heating of the cylinder allowed temperatures of $300{ }^{\circ} \mathrm{C}$ to be reached whereby the dye vapor was entrained in the flow of the host gas and the mixture deposited on a sapphire window at cryogenic temperatures (20 K typically). Gas flows in excess of $10 \mathrm{mmol} / \mathrm{h}$ were required to achieve isolation of monomer dye molecules.

Details of the cryogenic setup were described previously. ${ }^{12}$ Typical sample thicknesses were about $250 \mu \mathrm{m}$. Isolation conditions for the dye molecules in the matrix, as well as sample concentrations were estimated from absorption spectroscopy. ${ }^{1}$ Either DCM, Rh610 or Rh6G dye pumped with a frequency doubled pulsed YAG or a $\mathrm{XeCl}$ excimer laser were used for exciting either the $Q_{X}$ and $Q_{Y}$ bands of $\mathrm{H}_{2}$ TBP or the $Q$ band of ZnTBP. Fluorescence was analyzed with a HRS 600 Jobin-Yvon spectrometer and recorded on a fast iCCD (ANDOR, model DH-720) camera. All results were measured at $8 \mathrm{~K}$.

Before presenting and discussing our results we want to emphasize one point concerning sample preparation. The method used for introducing the guest molecules into the gas flow, then condensed as a solid on a low temperature substrate, makes it quite difficult to control the final guest concentration in the crystal host accurately. To ensure that we have worked on low concentration samples, thereby preserving the isolation of the guest molecules, we made absorption spectral measurements at different guest concentrations beforehand. A very useful indicator we have found in this regard is the width of absorption bands. In the case of aggregate formation absorption bands appeared quite broad and unstructured. ${ }^{13}$ Fluorescence and other experiments were then done on samples with the narrowest or highly structured bands.

\section{RESULTS AND DISCUSSION}

The results obtained for $\mathrm{H}_{2} \mathrm{TBP}$ isolated in $\mathrm{Ar}$, Xe, and $\mathrm{N}_{2}$ and for ZnTBP isolated in $\mathrm{Ar}$ and $\mathrm{N}_{2}$ are presented and discussed in this paper. 


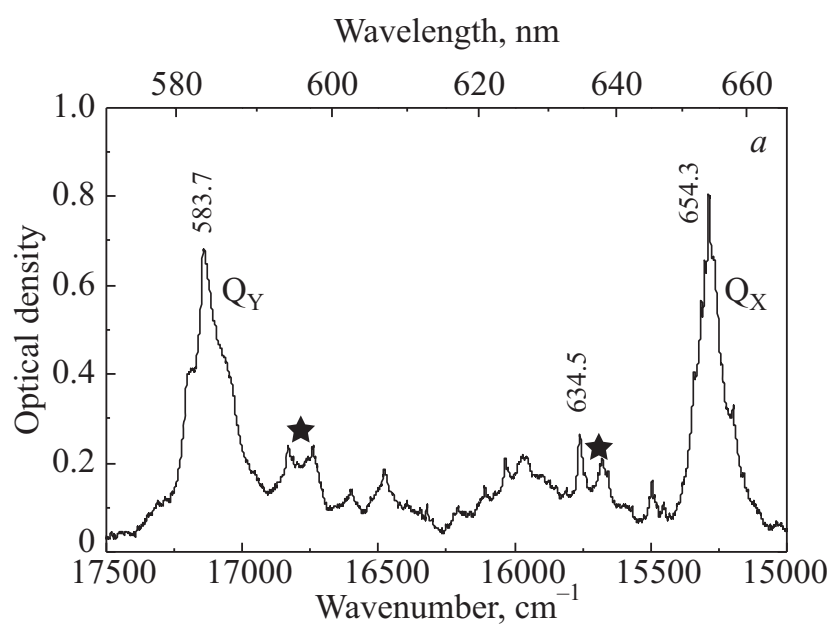

is important to mention that the values of the $Q_{X}-Q_{Y}$ splitting are calculated by simply taking the difference between the maximum of the intense $Q_{Y}$ band associated to the localization of the $S_{2} \leftarrow S_{0}$ transition. Indeed, this is not entirely true as in doing this, the Fermi resonance ${ }^{9,14}$ which influences the exact position and intensity of the $Q_{Y}(0-0)$ band is not considered here. Therefore the position as well as the intensity of the $Q_{Y}(0-0)$ band are not precisely defined in this work.

Another comparison with previously reported spectra of $\mathrm{H}_{2} \mathrm{Pc}$ in the same host allows us to make the following comments: absorption lines recorded for $\mathrm{H}_{2} \mathrm{Pc} / \mathrm{Ar}$ under similar conditions looked narrower that those reported in Fig. 3. The broadening observed is probably not due to a strong contribution from aggregates, even if we cannot completely exclude it. Indeed, $\mathrm{H}_{2} \mathrm{TBP} / \mathrm{Ar}$ spectra show some structured features that very likely arise from a greater number of separated sites in the matrix. From a more detailed view of the $Q_{X}$ absorption band of $\mathrm{H}_{2} \mathrm{TBP} / \mathrm{Ar}$, at least seven sites can be distinguished. This point will be more explicitly clarified in a later paragraph concerning the observation of spectral hole burning. Note that the presence of multiple sites would also explain the width of the bands.

The absorption spectrum of $\mathrm{ZnTBP} / \mathrm{Ar}$ at $8 \mathrm{~K}$ is shown in Fig. 3b. ZnTBP belongs to the $D_{4 h}$ point group, while $\mathrm{H}_{2}$ TBP belongs to $D_{2 h}$ and for symmetry reasons the two bands $Q_{X}$ and $Q_{Y}$ collapse into only one $Q$ band located at about $613 \mathrm{~nm}$ in the argon host, i.e. in between the positions of the $Q_{X}$ and $Q_{Y}$ bands of $\mathrm{H}_{2}$ TBP. From spectroscopic measurements of $\mathrm{ZnTBP}$ in supersonic expansion, ${ }^{15}$ the $S_{1}$ state was located at $16579 \mathrm{~cm}^{-1}(603.2 \mathrm{~nm})$ above the ground state, a value slightly blue-shifted compared to $16316 \mathrm{~cm}^{-1}$ $(612.9 \mathrm{~nm})$ found here at $8 \mathrm{~K}$. The present matrix value is in perfect agreement with the previously reported one as $16313 \mathrm{~cm}^{-1}$ for ZnTBP/Ar at $20 \mathrm{~K}^{2}$

While the absorptions in nitrogen matrices are quite similar to those in argon hosts, the absorption spectra of $\mathrm{H}_{2}$ TBP in Xe clearly exhibits «doublets» structures probably corresponding to two major sites. A comparison between the energy locations of the $S_{1} \leftarrow S_{0}$ and $S_{2} \leftarrow S_{0}$ transitions for $\mathrm{H}_{2}$ TBP and ZnTBP in different environments at low temperatures is presented in Table I. ecules. The two main bands appearing at about $654 \mathrm{~nm}$ $\left(15284 \mathrm{~cm}^{-1}\right)$ and $584 \mathrm{~nm}\left(17132 \mathrm{~cm}^{-1}\right)$ belong to absorptions of the split $Q$ band, frequently labeled as the $Q_{X}$ and $Q_{Y}$ bands, respectively, in order of increasing energy. The two corresponding bands in the $\mathrm{H}_{2} \mathrm{Pc} / \mathrm{Ar}$ absorption spectrum are located at about $677 \mathrm{~nm}\left(14771 \mathrm{~cm}^{-1}\right)$ and $635 \mathrm{~nm}$ $\left(15748 \mathrm{~cm}^{-1}\right)$. The shift of the absorption bands observed between $\mathrm{H}_{2}$ TBP and $\mathrm{H}_{2} \mathrm{Pc}$ results from the change in energy of the molecular orbitals due to the replacement of the interpyrrolic carbons $\mathrm{C}_{m}$ by the nitrogen atoms (Fig. 1). As a consequence, the $\mathrm{H}_{2} \mathrm{Pc} Q$ bands are shifted to the red while the $B$ bands in the Soret's region are oppositely blue shifted.

From an analysis of the different absorption spectra that we recorded for benzoporphyrin in several hosts we can extract the following results about the $Q_{X}-Q_{Y}$ splitting. For $\mathrm{H}_{2} \mathrm{TBP}$, we report values of about $1848 \mathrm{~cm}^{-1}, 1655 \mathrm{~cm}^{-1}$ (average value for the two main sites observed), and $1851 \mathrm{~cm}^{-1}$ for Ar, Xe and $\mathrm{N}_{2}$ hosts, respectively, as compared to the splitting values of $982 \mathrm{~cm}^{-1}, 958 \mathrm{~cm}^{-1}$, and

\section{B. Emission spectra}

\section{1. $\alpha$-fluorescence}

Singlet emission spectra recorded for $\mathrm{H}_{2} \mathrm{TBP}$ and ZnTBP in the Ar host are shown in Fig. 4. Both fluorescence spectra shown for $\mathrm{H}_{2} \mathrm{TBP}$ and $\mathrm{ZnTBP}$ are presented with the same horizontal scale with the position of the $0-0$ region made coincident to allow comparison of their vibronic structure. For $\mathrm{H}_{2}$ TBP the non-resonant fluorescence spectrum is shown (Fig. 4a). The higher energy band shown at about $654.2 \mathrm{~nm}$ corresponds to the $0-0$ zero-phonon origin band. Its structure suggests the presence of several marked sites as also noticed from the absorption spectrum. Besides the rather intense electronic origin region, the most intense band which appears in the $\mathrm{H}_{2} \mathrm{TBP} / \mathrm{Ar}$ spectrum is that centered at $731.9 \mathrm{~nm}$, revealing, like in the case of $\mathrm{H}_{2} \mathrm{Pc}$, the existence 
TABLE I. Experimental spectral positions of the $Q$ bands for $\mathrm{H}_{2} \mathrm{TBP}$ and ZnTBP in different environments.

\begin{tabular}{|c|c|c|c|c|}
\hline Guest & Host & Transition, band & $\begin{array}{c}\text { Peak energy } \\
\mathrm{cm}^{-1}(\mathrm{~nm})\end{array}$ & References \\
\hline \multirow[t]{10}{*}{$\mathrm{H}_{2} \mathrm{TBP}$} & \multirow[t]{2}{*}{ Ar, $8 \mathrm{~K}$} & $S_{1} \leftarrow S_{0}, Q_{X}$ & $15284(654.3)$ & \multirow[t]{2}{*}{ This work } \\
\hline & & $S_{2} \leftarrow S_{0}, Q_{Y}$ & $17132(583.7)$ & \\
\hline & \multirow{4}{*}{$\begin{array}{c}\mathrm{Xe}, 8 \mathrm{~K} \\
(2 \text { main sites })\end{array}$} & $S_{1}^{\prime} \leftarrow S_{0}, \mathrm{QX}$ & $14986(667.3)$ & \multirow[t]{4}{*}{ This work } \\
\hline & & $S_{2}^{\prime} \leftarrow S_{0}, \mathrm{QY}$ & $16631(601.3)$ & \\
\hline & & $S_{1}^{\prime \prime} \leftarrow S_{0}, \mathrm{QX}$ & $15205(657.7)$ & \\
\hline & & $S_{2}^{\prime \prime} \leftarrow S_{0}, \mathrm{QY}$ & $16869(592.8)$ & \\
\hline & \multirow[t]{2}{*}{$\mathrm{N}_{2}, 8 \mathrm{~K}$} & $S_{1} \leftarrow S_{0}, Q_{X}$ & $15305(653.4)$ & \multirow[t]{4}{*}{ This work } \\
\hline & & $S_{2} \leftarrow S_{0}, Q_{Y}$ & 17156 (582.9) & \\
\hline & \multirow[t]{2}{*}{$n-\mathrm{C}_{8}, 4.2 \mathrm{~K}$} & $S_{1} \leftarrow S_{0}, Q_{X}$ & $15175(659.0)$ & \\
\hline & & $S_{2} \leftarrow S_{0}, Q_{Y}$ & $16600(602.4)$ & \\
\hline \multirow[t]{5}{*}{ ZnTBP } & Ar, $8 \mathrm{~K}$ & $S_{1,2} \leftarrow S_{0}, \mathrm{Q}$ & 16316 (612.9) & This work \\
\hline & $\mathrm{N}_{2}, 8 \mathrm{~K}$ & $S_{1,2} \leftarrow S_{0}, \mathrm{Q}$ & $16329(612.4)$ & \multirow{4}{*}{$\begin{array}{c}\text { This work } \\
2 \\
6 \\
15\end{array}$} \\
\hline & Ar, $20 \mathrm{~K}$ & $S_{1,2} \leftarrow S_{0}, \mathrm{Q}$ & $16313(613)$ & \\
\hline & $n-\mathrm{C}_{8}, 4.2 \mathrm{~K}$ & $S_{1,2} \leftarrow S_{0}, \mathrm{Q}$ & $15954(626.8)$ & \\
\hline & $\begin{array}{l}\text { He, supersonic } \\
\text { expansion }\end{array}$ & $S_{1,2} \leftarrow S_{0}, \mathrm{Q}$ & $16579(603.2)$ & \\
\hline
\end{tabular}

of stimulated emission. Stimulated emission observed in the case of $\mathrm{H}_{2}$ TBP will be discussed in detail in a paragraph to follow.

The fluorescence emission from ZnTBP/Ar is shown in Fig. 4b. A complete analysis of this fluorescence spectrum is complicated by the probable presence of impurities. However, undoubtedly, the major band at $681.2 \mathrm{~nm}$ is assigned to the same active mode at approximately $1636 \mathrm{~cm}^{-1}$ which gives rise to stimulated emission in $\mathrm{H}_{2} \mathrm{TBP} / \mathrm{Ar}$. Nevertheless, no evidence of stimulated emission was observed in that case irrespective of the laser intensity. A negative result concerning the observation of stimulated emission was also obtained in the case of $\mathrm{ZnTBP} / \mathrm{N}_{2}$.

\section{2. $\beta$-stimulated emission}

As previously indicated the intense and narrowed line at $731.9 \mathrm{~nm}\left(13663 \mathrm{~cm}^{-1}\right)$ is due to stimulated emission. While collecting data about this stimulated emission we noticed a qualitatively dependence upon a threshold effect even if we did not characterize it quantitatively. This stimulated emission was also observed in $\mathrm{N}_{2}$ matrices around $731 \mathrm{~nm}$ under pulsed laser excitation of $Q_{X}$ and/or $Q_{Y}$, but not in Xe crystals. Such a situation is quite similar to what we reported already in the case of free-base phthalocyanine, for which stimulated emission was also observed in $\mathrm{Ne}, \mathrm{Ar}, \mathrm{Kr}$ but not in Xe hosts. ${ }^{1}$ Stimulated emissions in $\mathrm{H}_{2} \mathrm{Pc}$ were assigned to specific vibronic transitions involving a vibration mode at about $1550 \mathrm{~cm}^{-1}$ assigned to the stretching vibration $\mathrm{C}_{\alpha} \mathrm{N}$ bonds of the $\mathrm{C}-\mathrm{N}-\mathrm{C}$ bridges of the central ring, ${ }^{16,17}$ whereas the corresponding mode in the case of $\mathrm{H}_{2}$ TBP is around $1620 \mathrm{~cm}^{-1}$. As was noted in Refs. 9 and 18 the $1620 \mathrm{~cm}^{-1}$ vibration of $\mathrm{H}_{2} \mathrm{TBP}$ corresponds to the $A_{g}$ stretching vibration of the $\mathrm{C}_{\alpha} \mathrm{C}_{m}$ bonds, i.e. to the vibration which is localized in the $\mathrm{C}_{\alpha} \mathrm{C}_{m} \mathrm{C}_{\alpha}$ bridges in $\mathrm{H}_{2} \mathrm{TBP}\left(\mathrm{C}_{\alpha}\right.$-carbon atom in the $\alpha$-position of the pyrrole rings, $\mathrm{C}_{m}$ - carbon atom in the meso- position $\left(\mathrm{C}_{\alpha} \mathrm{C}_{m} \mathrm{C}_{\alpha}\right.$ bridges $)$ as shown in Fig. 1).

When the pump power intensity was increased by a fac$\left.\mathbf{s}^{\sim f} i^{h} \cdots+\mathbf{i}\right\rangle$, from tens of $\mu \mathrm{J} /$ pulse to hundreds of

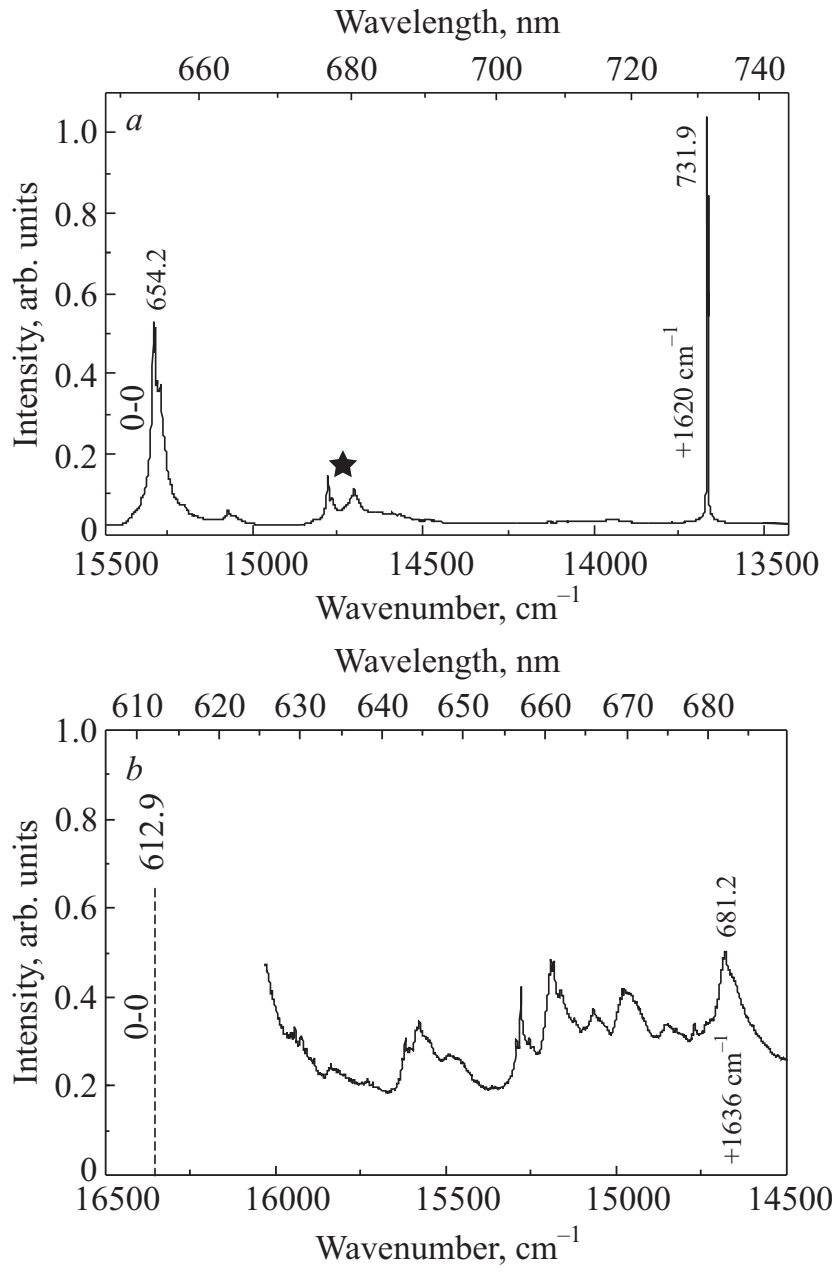

FIG. 4. Fluorescence spectrum of free-base $\mathrm{H}_{2}$ TBP recorded under nonselective laser excitation at about $583 \mathrm{~nm}$ (a). Fluorescence spectrum of ZnTBP under resonant excitation wavelength at $612.9 \mathrm{~nm}$ (b). Both fluorescence spectra were obtained with Ar as the host crystal at about $8 \mathrm{~K}$. The horizontal scales have been shifted adequately to make coincident the singlet-singlet electronic origin regions. The asterisk indicates the fluorescence line due to residual free-base phthalocyanine $\mathrm{H}_{2} \mathrm{Pc}$.

$\mu \mathrm{J} /$ pulse, the emission band at about $732 \mathrm{~nm}$ gained enormously in intensity while the others remained unchanged or diminished slightly. Moreover, the linewidth of this emission band was reduced considerably while its intensity increased. The width of the band is approaching that of the exciting laser, decreasing from approximately $8 \mathrm{~cm}^{-1}$ to $2 \mathrm{~cm}^{-1}$, a value surely limited by the resolving power of the monochromator.

Figure 5 shows a two-dimensional (2D) excitation/ emission plot of the stimulated emission observed for freebase $\mathrm{H}_{2}$ TBP isolated in solid Ar at $8 \mathrm{~K}$. The plot in Fig. 5a depicts the sites emission detected with excitation in the 0-0 region of the $S_{1} \leftarrow S_{0}$ transition $\left(Q_{X}\right.$ band) while Fig. $5 \mathrm{~b}$ depicts that produced with excitation of the $S_{2} \leftarrow S_{0}$ transition ( $Q_{Y}$ band). Both $2 \mathrm{D}$ excitation/emission plots were recorded while monitoring the SE mode centered around $732 \mathrm{~nm}$. For $\mathrm{H}_{2} \mathrm{TBP} / \mathrm{Ar}$ under pulsed excitation in the $Q_{X}$ region, around $654 \mathrm{~nm}$, doublet SE lines (separated by $3 \mathrm{~cm}^{-1}$ ) were recorded giving rise to similar excitation spectra (separated by about $5 \mathrm{~cm}^{-1}$ ).

An analysis of the SE signals generated under selective excitation at $654.43 \mathrm{~nm}$ and $654.25 \mathrm{~nm}$ inside the broad $Q_{X}$ 

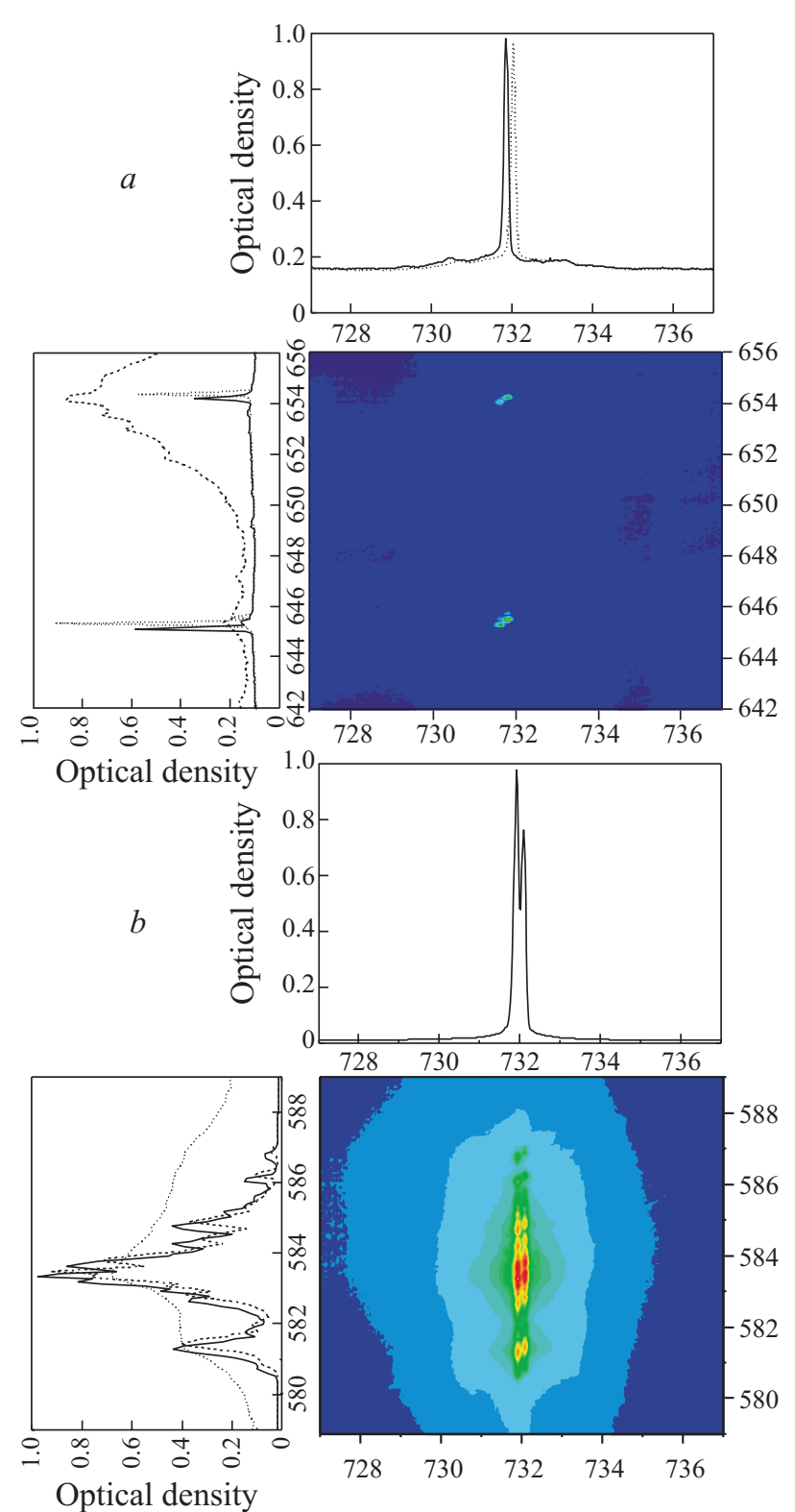

FIG. 5. 2D emission-excitation spectra of free-base $\mathrm{H}_{2} \mathrm{TBP} / \mathrm{Ar}$ at $8 \mathrm{~K}$ recorded under selective laser excitation. Both $Q_{X}$ and $Q_{Y}$ regions were studied, emission spectra are shown on top, excitation spectra, together with the absorption spectra (dotted lines) are on the left. Analysis of the 2D spectrum under SE conditions of $Q_{X}$ region around $654 \mathrm{~nm}$ (a). Analysis of the 2D spectrum under SE conditions of $Q_{Y}$ region around $584 \mathrm{~nm}$ (b).

absorption band of $\mathrm{H}_{2} \mathrm{TBP} / \mathrm{Ar}$ at $8 \mathrm{~K}$ revealed the existence of two stimulated emissions, which are assigned to two distinct sites (Fig. 5a). These two sites look independent. In contrast to the case of $\mathrm{H}_{2} \mathrm{Pc}$, no phonons are excited but a vibration at about $215 \mathrm{~cm}^{-1}$ from the origin band is excited, as evidenced by looking at the absorption spectrum (dotted line) shown superimposed to the excitation spectra. Clearly, excitation spectra recorded for the SE signal provide greatly enhanced spectroscopic information over what can be extracted from either conventional fluorescence excitation or absorption spectroscopy.

The situation is more complicated in the case of $\mathrm{H}_{2} \mathrm{TBP} / \mathrm{N}_{2}$ (not shown). Indeed, four sites giving rise to $\mathrm{SE}$ signals at $13678 \mathrm{~cm}^{-1}(731.1 \mathrm{~nm}), 13687 \mathrm{~cm}^{-1}(730.6 \mathrm{~nm})$, $13694 \mathrm{~cm}^{-1}(730.3 \mathrm{~nm})$ and $13701 \mathrm{~cm}^{-1}(729.9 \mathrm{~nm})$ were

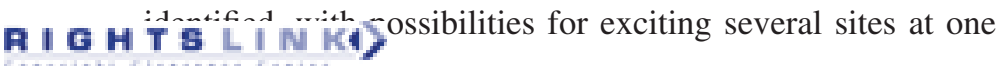

time because of possible transfers from one site to another as reported already for $\mathrm{H}_{2} \mathrm{Pc}$. ${ }^{1}$ In this case the excitation spectra of SE lines exhibit quite intense phonon bands allowing the simultaneous excitation of all sites.

Upon excitation of the $Q_{Y}$ band of $\mathrm{H}_{2} \mathrm{TBP} / \mathrm{Ar}$ the observation of two sites is still revealed by the occurrence of stimulated emission, but the excitation spectra recorded were found to be more complex due to vibronic coupling between vibrational levels from the lower $Q_{X}$ state and the electronic $Q_{Y}$ state. While monitoring one or the other site, the corresponding excitation spectra were revealed, demonstrating that they have the same structure simply shifted with respect to the one another by $\sim 4 \mathrm{~cm}^{-1}$, the exact energy spacing between the two sites. From this important observation we can conclude that the vibronic coupling between $Q_{X}$ and $Q_{Y}$ is quite similar for the two sites, and that no phonon modes are contributing (Fig. 5b). For $Q_{Y}$ in the case of $\mathrm{H}_{2} \mathrm{TBP} / \mathrm{N}_{2}$, the situation (also not shown) needs to be clarified as disturbances due to excitation of phonon modes from the matrix are also assumed to contribute. Under $Q_{Y}$ excitation, only the two main SE lines located at $13678 \mathrm{~cm}^{-1}(731.1 \mathrm{~nm})$ and $13687 \mathrm{~cm}^{-1}(730.6 \mathrm{~nm})$ are clearly observed, allowing the identification of two main sites separated by about $\sim 9 \mathrm{~cm}^{-1}$.

In the case of the fluorescence spectra of ZnTBP/Ar under pulsed laser excitation no stimulated emission enhancement could be observed irrespective of the incident laser intensity. A similar observation was also reported for $\mathrm{H}_{2} \mathrm{TBP} / \mathrm{Xe}$. A possible explanation of the absence of stimulated emission in the case of $\mathrm{ZnTBP}$ and $\mathrm{H}_{2} \mathrm{TBP} / \mathrm{Xe}$ could be the heavy atom ( $\mathrm{Zn}$ or $\mathrm{Xe}$ ) effect which increases the probability of the intersystem crossing rate from the excited singlet $S_{1}$ state level to the lower lying triplet $T_{1}$ state. This non-radiative relaxation mechanism then competes with the fluorescence and thereby reduces the chance of the excited singlet state population for reaching the threshold condition. As a consequence of the enhanced intersystem crossing the triplet state is efficiently populated, leading to the observation of a phosphorescence signal.

\section{3. c-phosphorescence}

In the case of ZnTBP/Ar, for which no stimulated emission could be monitored, a rather strong phosphorescence signal was recorded with a lifetime longer than $30 \mathrm{~ms}$. The corresponding phosphorescence spectrum is shown in Fig. 6.

The recorded spectrum is not easily understandable, and the situation is even unclear after attempting to analyze the associated excitation spectra (not shown). However, inspection of Fig. 6 shows that a «doublet» structure is clearly apparent in the phosphorescence spectra of ZnTBP/Ar and $\mathrm{ZnTBP} / \mathrm{N}_{2}$. A similar «doublet» structure was earlier reported for $\mathrm{ZnTBP} / n-\mathrm{C}_{8}$ by Arabei et al. ${ }^{6}$ The interpretation given by the authors was as follows: due to non-correlation between $S_{1}-S_{0}$ and $T_{1}-T_{0}$ transition energies in the site structure, a site selective excitation in the $S_{1}-S_{0}$ region can induce the phosphorescence from different sites with distinct $T_{1}-S_{0}$ transition energies. However several questions remain unsolved still and we consider that the phosphorescence spectra of ZnTBP in various cryogenic matrices requires further analysis. Amongst these, it is necessary to clarify whether or not efficient intersystem transfer, as ascertained 


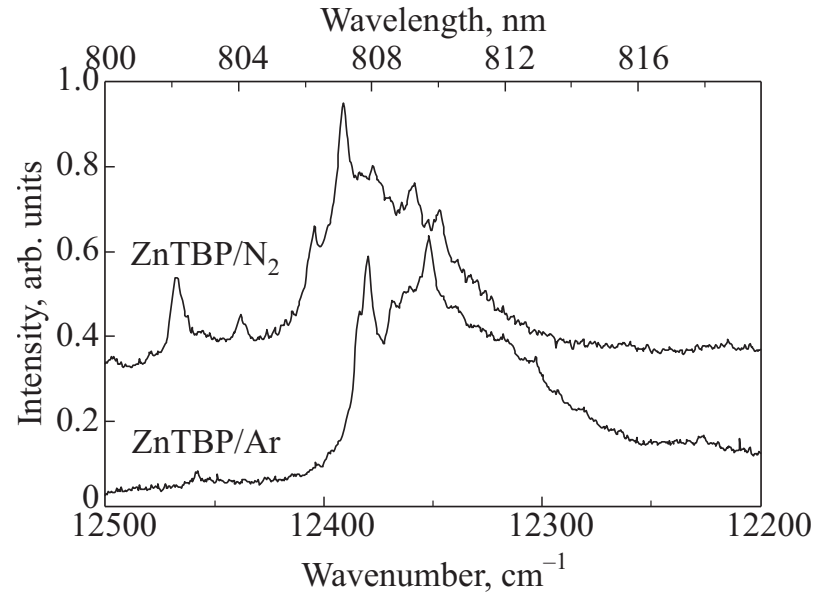

FIG. 6. Phosphorescence spectra of $\mathrm{ZnTBP} / \mathrm{Ar}$ and $\mathrm{ZnTBP} / \mathrm{N}_{2}$ recorded under selective laser excitation at about $612 \mathrm{~nm}$. The spectra were recorded at about $8 \mathrm{~K}$.

by the observed phosphorescence, is the major factor for the non-observation of stimulated emission in ZnTBP.

\section{Spectral hole-burning}

With $\mathrm{H}_{2} \mathrm{TBP} / \mathrm{Ar}$ persistent spectral changes could be detected under selective laser excitation at $8 \mathrm{~K}$. These spectral hole-burning changes were observed when looking for stimulated emission conditions under selective excitation of one or the other site. At low temperature the spectral holeburning phenomenon is quite well known in the case of freebase porphyrins. ${ }^{19}$ It is then not unusual to observe it with $\mathrm{H}_{2}$ TBP. Figure 7 shows an expanded view of the absorption spectrum corresponding to the $Q_{X}$ absorption band before excitation and then, after excitation of one site and then of the other one. The inset of Fig. 7 presents a more expanded view to show the changes observed close to the band maximum. Typical burning times needed to observe this persistent spectral change were in the order of one hour for laser energy of $\sim 40 \mu \mathrm{J} /$ pulse at $10 \mathrm{~Hz}$.

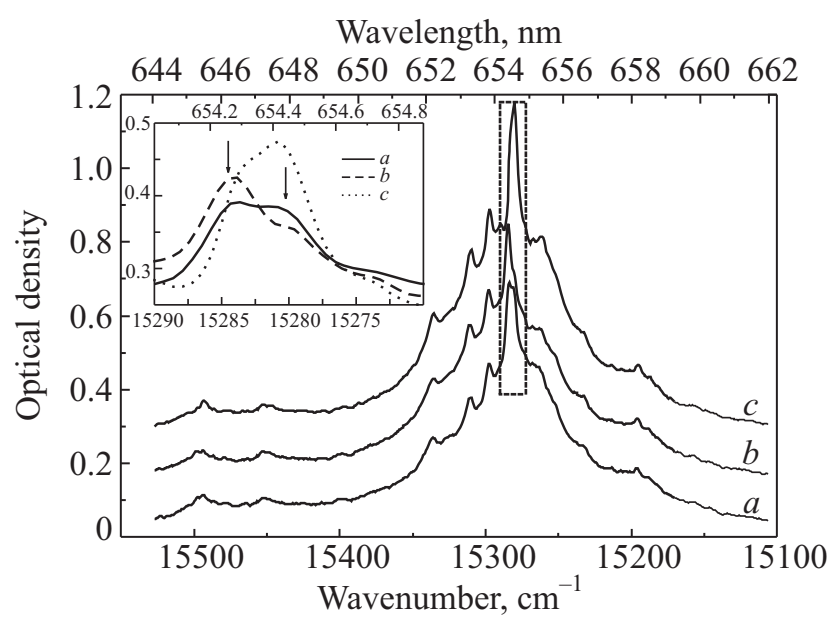

FIG. 7. Extended view of the absorption spectrum corresponding to the $Q_{X}$ absorption band of $\mathrm{H}_{2} \mathrm{TBP} / \mathrm{Ar}$ at $8 \mathrm{~K}$. a, b and $\mathrm{c}$ are the traces recorded before irradiation, after irradiation at $654.43 \mathrm{~nm}$ and after irradiation at $654.25 \mathrm{~nm}$. Inset: evidence of persistent spectral changes (hole-burning efA $\left.1 \mathbf{G ~} \mathbf{H} \mathbf{T}_{\mathbf{S}} \mathbf{L} 1 \mathbf{N} \mathbf{K}\right)$ cific sites in the $Q_{X}$ origin region.
Among the different sites which are spectrally identified in the absorption, only two specific lines showed selective persistent spectral changes. Burning at $654.25 \mathrm{~nm}$ induced a decrease of the absorption at this wavelength, while a similar change was observed when burning the other site at $654.43 \mathrm{~nm}$. It is interesting to notice that quite similar spectral changes are observed on the vibronic band located at about $15500 \mathrm{~cm}^{-1}$, assigned to the same vibration mode as the one which gives rise to SE under laser excitation. This phenomenon could be explained by tautomerization. In this model the two sites would be associated with the two tautomers of the porphyrin molecule, and the observed changes arise from the interconversion of one form to another, i.e. the flipping of the inner protons from one position to the other. ${ }^{20,21}$ In the case of $\mathrm{H}_{2} \mathrm{TBP} / \mathrm{Ar}$ this process has a rather low efficiency and cannot reduce SE significantly. Such a situation differs from what we reported for $\mathrm{H}_{2} \mathrm{Pc} / \mathrm{Ne}$, ${ }^{1}$ for which very efficient hole-burning phenomenon was observed in the 0-0 $S_{1} \leftarrow S_{0}$ absorption band with even weak dye laser excitation. Consequently, as the excited family of sites disappeared during excitation, SE was not sustainable for long periods in this solid.

\section{CONCLUSIONS}

The results obtained in the present work confirm that the phenomenon of stimulated emission first observed for phthalocyanines in cryogenic matrices under modest conditions of pulsed laser excitation is not an exception. With free-base $\mathrm{H}_{2}$ TBP stimulated emission was observed in $\mathrm{Ar}$ and $\mathrm{N}_{2}$ matrices but not in $\mathrm{Xe}$ matrices. The vibronic transition involved is, like in the case of $\mathrm{H}_{2} \mathrm{Pc}$, the one involving a mode at about $1620 \mathrm{~cm}^{-1}$, a particularly active mode corresponding to the stretching $A_{g}$ vibration of the $\mathrm{C}_{\alpha} \mathrm{C}_{m}$ bonds. ${ }^{9,18} \mathrm{We}$ also reported the observation of persistent spectral holeburning; however the quite low efficiency of the burning process can not affect SE. Finally, no stimulated emission was recorded with $\mathrm{ZnTBP}$, but the appearance of rather strong phosphorescence was noticed. From all results we have obtained on $\mathrm{SE}$ in cryogenic matrices with $\mathrm{H}_{2} \mathrm{Pc}, \mathrm{ZnPc}$, as well as $\mathrm{H}_{2}$ TBP we can reasonably predict that SE should also be observable for these molecules isolated in other solid materials that also produce narrow linewidths such as Shpol'skii matrices, like $n$-octane. Other possible interesting candidates could also be sol-gel or polymer hosts.

Another important remark is the following: compared to what is obtained in normal fluorescence excitation scans, greatly enhanced site selectivity is obtained for excitation scans recorded for the SE mode. This behavior stems from the increased resolution of individual sites whose lineshape change from featureless fluorescence excitation bands to a highly structured SE excitation band. The very well-resolved spectra available in SE excitation scans allow detailed exploration of site occupancies in a given solid and from one solid to another. Added to our previous, already published results, ${ }^{1}$ the results of the present study of SE should allow new insights into site effects and site selectivity.

\section{ACKNOWLEDGMENTS}

The assistance of Dr. Michel Broquier and Dr. Pierre Çarçabal for the design of the oven used for vaporization of 
the porphyrin molecules is gratefully acknowledged. The France-Belarus cooperation was supported by the CNRS/ BRFFR No. 23181 agreement and grant BRFFR No. F09F001. McC's participation in this work was made possible with support from Science Foundation Ireland (SFI), Research Frontiers Programme (06/RFP/CHP012).

${ }^{a)}$ Email: jean-pierre.galaup@lac.u-psud.fr

${ }^{1}$ N. Dozova, C. Murray, J. G. McCaffrey, N. Shafizadeh, and C. Crépin, Phys. Chem. Chem. Phys. 10, 2167 (2008).

${ }^{2}$ L. Bajema, M. Gouterman, and C. B. Rose, J. Mol. Spectrosc. 39, 421 (1971).

${ }^{3}$ L. Bajema, M. Gouterman, and B. Meyer, J. Mol. Spectrosc. 27, 225 (1968).

${ }^{4}$ A. Starukhin, A. Shulga, and J. Waluk, Chem. Phys. Lett. 272, 405 (1997).

${ }^{5}$ A. Kyrychenko and J. Waluk, J. Chem. Phys. 123, 064706 (2005); ibid., references.

${ }^{6}$ S. M. Arabei, K. N. Solov'ev, S. F. Shkirman, and T. F. Kachura, J. Appl. Spectrosc. 50, 606 (1989).

${ }^{7}$ S. M. Arabei, J.-P. Galaup, K. N. Solovyov, and V. F. Donyagina, Chem. Phys. 311, 307 (2005).

${ }^{8}$ S. M. Arabei, K. N. Solov'ev, and T. F. Kachura, Opt. Spectrosc. 65, 213 (1988); S. M. Arabei, K. N. Solov'ev, and A. E. Turkova, Opt. Spectrosc.
80, 379 (1996)

${ }^{9}$ S. M. Arabei, K. N. Solovyov, and Yu. I. Tatulchenkov, Opt. Spectrosc. 73, 406 (1992)

${ }^{10}$ S. M. Arabei, J. Appl. Spectrosc. 57, 572 (1992).

${ }^{11}$ R. P. Linstead and F. T. Weiss, J. Chem. Soc. 11, 2975 (1950).

${ }^{12}$ C. Gée, A. Cuisset, L. Divay, and C. Crépin, J. Chem. Phys. 116, 4993 (2002).

${ }^{13}$ B. J. Prince, B. E. Williamson, and R. J. Reeves, J. Lumin. 93, 293 (2001).

${ }^{14}$ S. M. Arabei, V. A. Kuzmitsky, and K. N. Solovyov, Opt. Spectrosc. 102, 692 (2007); S. M. Arabei, V. A. Kuzmitsky, and K. N. Solovyov, Chem. Phys. 352, 197 (2008).

${ }^{15}$ U. Even, J. Magen, J. Jortner, and J. Friedman, J. Chem. Phys. 77, 4384 (1982).

${ }^{16}$ D. R. Tackley, G. Dent, and W. E. Smith, Phys. Chem. Chem. Phys. 3, 1419 (2001).

${ }^{17}$ C. Murray, N. Dozova, J. G. McCaffrey, N. Shafizadeh, and C. Crépin, manuscript in preparation.

${ }^{18}$ S. F. Shkirman, L. L. Gladkov, V. K. Kostantinova, and K. N. Solov'ev, Spectrosc. Lett. 31, 1749 (1998); S. F. Shkirman, L. L. Gladkov, V. K. Kostantinova, and K. N. Solov'ev, J. Appl. Spectrosc. 66, 400 (1999).

${ }^{19} \mathrm{~J}$.-P. Galaup in Advances in Multiphoton Processes and Spectroscopy, edited by S. H. Lin, A. A. Villaeys, and Y. Fujimura, World Scientific Publishing Co Pte Ltd, Singapore, September, Vol. 16 (2004), p. 73.

${ }^{20}$ K. N. Solovyov, I. E. Zalesskii, V. N. Kotlo, and S. F. Shkirman, JETF Lett. 17, 332 (1973).

${ }^{21}$ J. G. Radziszewski, J. Waluk, and J. Michl, Chem. Phys. 136, 165 (1989).

This article was published in English in the original Russian journal. Reproduced here with stylistic changes by AIP. 